\title{
A neuropsychological evaluation of orthorexia nervosa*
}

\author{
Nancy S. Koven ${ }^{1 \#}$, Rina Senbonmatsu ${ }^{2}$ \\ ${ }^{1}$ Department of Psychology, Bates College, Maine, USA \\ ${ }^{2}$ School of Medicine, Semmelweis University, Budapest, Hungary \\ Email: $\underline{\text { nkoven@ bates.edu }}$
}

Received 4 January 2013; revised 6 February 2013; accepted 15 February 2013

Copyright (C) 2013 Nancy S. Koven, Rina Senbonmatsu. This is an open access article distributed under the Creative Commons Attribution License, which permits unrestricted use, distribution, and reproduction in any medium, provided the original work is properly cited.

\begin{abstract}
Orthorexia nervosa (ON) describes a fixation for proper nutrition and healthy foods that leads to dietary restrictions and nutritional deficiencies over time. Given symptom overlap among $\mathrm{ON}$, obsessive-compulsive disorder (OCD), and anorexia nervosa (AN), this study examines whether $\mathrm{ON}$ has a unique neuropsychological profile. Participants completed symptom checklists and neuropsychological tests chosen for their sensitivity to AN and OCD. Results showed that, across the full sample, $\mathrm{ON}$ is independently associated with self-reported weaknesses in Set-Shifting, Emotional Control, Self-Monitoring, and Working Memory. After controlling for AN and OCD variables, high- and low-ON groups differed in total correct trials on the Wisconsin Card Sorting Test. These areas of executive functioning, which are explained in the context of ON symptomatology, represent what would be considered the neuropsychological overlap among OCD and AN profiles.
\end{abstract}

Keywords: Orthorexia Nervosa; Neuropsychology; Executive Function; Anorexia Nervosa; Obsessive Compulsive Disorder

\section{INTRODUCTION}

Derived from the Greek orthos (proper) and orexia (appetite), orthorexia nervosa (ON) describes a set of eatingrelated behaviors that include a fixation to eat healthy, biologically-pure foods and an inflated expectation about the personal benefits of healthy eating. Orthorexic individuals typically avoid food that has been treated with herbicides, pesticides, or artificial substances, and they worry excessively about the techniques and materials used in food preparation. This cautious eating style leads

\footnotetext{
"The authors have no conflicts of interest to declare.

\#Corresponding author.
}

to omission of food groups and eventually to nutritional deficiencies; the intense fixation on diet results in a loss of social relationships and impoverished quality of life [1]. To date, research has focused on the emotional and physical outcomes of ON, with little attention to cognitive mechanisms. There is a reason to expect cognitive correlates of $\mathrm{ON}$, however, as $\mathrm{ON}$ resembles two other psychiatric conditions, anorexia nervosa (AN) and obsessive-compulsive disorder (OCD).

At the symptom level, ON and AN share characteristics such as tendencies toward perfectionism, high comorbid anxiety, and need for control [2]. Like individuals with AN, those with $\mathrm{ON}$ consider the ability to follow a restrictive diet to be an achievement of self-discipline and perceive deviation from that diet as a failure of self-control [1]. While self-starvation is seen in both conditions [3], the ultimate goals of patients are different; orthorexic individuals want to improve and/or manage their health via diet, whereas anorexic individuals are concerned with physical body image as driven by fear of obesity. While anorexics shamefully hide their habits, orthorexics tend to flaunt their habits [1]. In addition to AN traits, orthorexic individuals manifest obsessivecompulsive tendencies, such as carefully weighing and measuring food, extreme meal planning, and intrusive thoughts of food outside of meal time [1]. Similar to OCD, ON interferes with the individual's normal routines and social interactions [3]. However, whereas the obsessions in OCD are perceived as ego-dystonic, the food-related thought content of individuals with $\mathrm{ON}$ is perceived as normal and appropriate.

Given symptom overlap across the three conditions, it is possible that individuals with ON possess similar cognitive traits as individuals with AN and/or OCD. Previous studies of AN have identified impairments in attention, verbal long-term memory, visuospatial functioning, and executive functioning (e.g., working memory, response inhibition, and cognitive flexibility) $[4,5]$. Individuals with OCD have difficulty with cognitive flexibil- 
ity [6] and response inhibition [7]. The goal of this study is to determine whether orthorexic individuals experience the same cognitive problems using standardized neuropsychological tests sensitive to performance differences in these domains. A nonclinical sample of young adults was chosen to minimize the effects of confounding psychiatric and neurological variables beyond those of interest. Recognizing that $\mathrm{ON}, \mathrm{AD}$, and $\mathrm{OCD}$ symptoms may co-occur even at subclinical thresholds, we obtained self-report indices of all three constructs in order to determine whether ON contributes unique variance to cognitive dysfunction. Given reports of adverse functional outcomes in ON [1], we expected cognitive dysfunction to covary with ON symptom severity and that individuals with $\mathrm{ON}\left(\mathrm{ON}^{+}\right)$would perform worse than those without $\left(\mathrm{ON}^{-}\right)$in one or more cognitive domains, independent of AN and OCD.

\section{METHOD}

\subsection{Participants}

The sample consisted of 100 right-handed young adults (79 women) who were recruited from undergraduate college courses in the Northeastern United States and who received course credit for participation. Participants ranged in age from 18 to 22 years $(M=19.3, S D=1.2)$, with $81 \%$ reporting their ethnicity as White/Caucasian, $15 \%$ as Asian/Asian-American, 3\% as Latino/Hispanic, and $1 \%$ as Black/African/African-American. Written informed consent was obtained per participant, and the protocol was approved by the Institutional Review Board. Rule-out criteria included English as a non-primary language, diagnosed psychiatric illness and/or history of treatment, history of neurological illness or brain injury, medical conditions and/or prescriptions known to have cognitive or emotional sequelae, and recent use of illicit substances known to affect cognition or emotion. Psychiatric screening was done with the Mini International Neuropsychiatric Interview (M.I.N.I.) English Version 5.0.0 [8].

\subsection{Procedure}

Participants completed the orthorexia scale (ORTO-15) [9], two subscales from the Eating Disorder Inventory, Second Edition (EDI-2) [10], and the Obsessive Compulsive Inventory, Revised (OCI-R) [11]. Participants then completed a neuropsychological battery that included tests in the following order: the Wide Range Achievement Test, Fourth Edition (WRAT-4) [12] Reading subtest, the Behavior Rating Inventory of Executive Functioning, Adult version (BRIEF-A) [13], the Trail Making subtest from the Delis-Kaplan Executive Function System (D-KEFS) [14], the acquisition and shortdelay recall trials of the California Verbal Learning Test,
Second Edition (CVLT-II) [15], the D-KEFS Design Fluency Test, the D-KEFS Color-Word Interference Test, the D-KEFS Verbal Fluency Test, the Wisconsin Card Sorting Test (WCST) [16], and the long-delay recall and recognition trials of the CVLT-II.

\subsection{Symptom Measures}

The ORTO-15 has 15 items scored on a 4-point scale that assess attitudes toward selecting and purchasing food (e.g., "Do you think that the food sold at the food store is unhealthy?" and "Are you willing to spend more money to have healthier food?"), habits of food consumption (e.g., "At present, do you eat meals alone?" and "Do you feel guilty when transgressing from a healthy diet?"), expectations about the effects of eating healthy food (e.g., "Do you think that eating only healthy food increases self-esteem?" and "Do you think that consuming healthy food improves your appearance?"), and the degree to which dietary concerns interfere with daily life (e.g., "Do you worry about food for more than three hours a day?" and "In the last three months, did the thought of food worry you?"). Total ON score is derived by summing across items, with scores below 40 indicative of ON [9]. Given its recent development, the psychometric properties of the ORTO-15 are largely uncharacterized, with only one validation study completed to date in an American college student sample [17]. In the current sample, the Cronbach alpha coefficient was 0.83 , suggesting adequate internal consistency.

The OCI-R, used to measure OCD symptom frequency, has 18 items divided into 6 subscales: Checking, Hoarding, Neutralizing, Obsessing, Ordering, and Washing. Items are scored on a five-point scale with higher values indicating greater dysfunction. The Cronbach alpha coefficient was 0.91 .

The EDI-2 includes 91 items that factor into 11 subscales: Drive for Thinness, Bulimia, Body Dissatisfaction, Ineffectiveness, Perfectionism, Interpersonal Distrust, Interoceptive Awareness, Maturity Fears, Asceticism, Impulse Regulation, and Social Insecurity. Of these, the Drive for Thinness, Body Dissatisfaction, and Perfectionism subscales capture the features of AN that most overlap with obsessive-compulsiveness [18]. However, in an effort to reduce the number of variables in the study, we excluded the Drive for Thinness scale on the grounds that its content is less relevant to $\mathrm{ON}$ than the other two EDI-2 subscales and given previous research indicating that many anorexic individuals do not endorse a drive for thinness $[19,20]$. We retained the Body Dissatisfaction and Perfectionism subscales, each of which uses a six-point scale and for which higher scores indicate greater severity. Cronbach alpha coefficients were 0.95 and 0.79 , respectively. 


\subsection{Neuropsychological Measures}

Baseline intellectual functioning was estimated using the WRAT-4 Reading subtest [12], in which participants read aloud words printed on a stimulus card. The raw score is converted to a standard score $(M=100, S D=$ $15)$, with higher scores indicating better word recognition and, thus, higher baseline intelligence.

The BRIEF-A is a 75-item measure that evaluates the experience of executive dysfunction in daily life [13]. Items, which are scored on a three-point scale, cluster to form nine subscales: Working Memory, Ability to Plan/ Organize, Ability to Task Monitor, Ability to Organize Materials, Ability to Inhibit, Task Initiation, Shifting, Emotional Control, and Self-Monitoring. Items are further summed into two higher-order scales, the Behavioral Regulation and Meta-Cognition Indices, which together comprise the Global Executive Composite. All subscales are normed for age and gender and reported as $T$-scores $(M=50, S D=10)$. Higher $T$-scores reflect greater dysfunction.

The CVLT-II is a measure of verbal learning and memory. The task involves orally administering a list of words to the participant across five learning trials and assessing memory in several ways. Variables of interest include the acquisition score, short- and long-delay free and cued recall scores, long-delay recognition score, and scores that reflect the use of serial and semantic clustering learning strategies. Scores are normed to $T$-scores $(M$ $=50, S D=10$ ), with higher $T$-scores indicating better performance [15].

The Wisconsin Card Sorting Test, Computer Version 4 (WCST:CV4) is a computerized assessment of abstract thinking, cognitive flexibility, set-shifting, and selfmonitoring. The participants are asked to match cards to one of four target stimuli based on color, shape, or number without being told the explicit rule by which to make the match. Variables of interest include the total number of trials, total correct responses, and the learning-to-learn index. Scores are standardized into $T$-scores $(M=50, S D$ $=10)$ or $z$-scores $(M=0, S D=1)$ based on available norms [16].

The Color-Word Interference Test is a four-part measure of cognitive flexibility and response inhibition. The first and second conditions require the participant to identify stimuli ink colors and to read aloud color-related words, respectively. In the third condition, the participant must name the ink color of a word whose semantic meaning is incongruent with the ink color itself. In the fourth condition, individuals must shift flexibly between word-reading and color-naming depending on the stimulus, thereby demonstrating both cognitive flexibility and response inhibition. Reaction time scores are converted to scaled scores $(M=10, S D=3)$ based on available norms [14].
The Trail Making Test is a five-part test that assesses processing speed and cognitive flexibility [14]. In the condition of interest (Number-Letter Switching), the participant must connect numbers and letters on a sheet of paper in alternating fashion. The reaction time score is converted to a scaled score $(M=10, S D=3)$ based on available norms [14].

Verbal Fluency is a three-part test to evaluate controlled retrieval, semantic memory, and processing speed. In the Letter Fluency task, the individual generates as many words as possible that begin with a designated letter within a set time frame. In the Category Fluency task, the individual generates words belonging to pre-designated categories within a set time frame. In the Category Switching task, the individual generates words belonging to two different categories within a set time frame while alternating between categories. Key variables, which include the number of unique responses per condition and set-shifting accuracy, are standardized to scaled scores $(M=10, S D=3)$ based on available norms [14].

Design Fluency is a three-part test of nonverbal fluency in which the individual creates unique designs by connecting dots on a sheet of paper. In the first two conditions, the participant connects filled dots and empty dots, respectively. In the third condition, the participant alternates connections to empty and filled dots, thereby requiring set-shifting in addition to fluency. Key variables are similar to those in Verbal Fluency [14].

\subsection{Statistical Analysis}

Using SPSS Version 20 for Windows, we utilized two approaches to data analysis. The first employed correlation and regression analyses using the full sample. We used Pearson correlations to determine the relationships between ORTO-15 score, other symptom variables, and neuropsychological variables. We then used hierarchical multiple regression to assess the ability of ORTO-15 score to predict neuropsychological performance after controlling for Body Dissatisfaction, Perfectionism, and OCI-R total scores. In the second approach, we identified subsets of participants who scored in the high and low range of the ORTO-15, respectively, and used analysis of variance (ANOVA) to determine whether endorsement of AN and OCD symptoms and cognitive test performance differ as a function of $\mathrm{ON}$ status. We then used analysis of covariance (ANCOVA) to assess whether inter-group differences in neuropsychological performance remain after controlling for Body Dissatisfaction, Perfectionism, and OCI-R total scores. Given that relationships between $\mathrm{ON}$ and cognition have not been established previously, we chose not to apply a Bonferroni correction for multiple comparisons, as adjusting the alpha significance threshold might inappropriately increase the possibility of missing a real effect [21]. 


\section{RESULTS}

\subsection{Analyses across the Full Sample}

Descriptive statistics for all variables are presented in Table 1. Pearson correlations (two-tailed) indicated that Body Dissatisfaction, perfectionism, and OCD symptoms increased as ORTO-15 score decreased (i.e., as orthorexia severity increased). Among OCI-R subscales, ORTO-15 correlated with Checking, $r=-0.34, p=0.001$, Neutralizing, $r=-0.34, p<0.001$, Obsessing, $r=-0.36$, $p<0.001$, Ordering, $r=-0.30, p=0.002$, and Washing, $r=-0.28, p=0.006$, but had no association with Hoarding, $r=-0.19, p=$ ns. Similarly, as ORTO-15 score decreased, self-reported executive dysfunction increased globally (Global Executive Composite, $r=-0.42, p<$ $0.001)$ as well as within behavior regulation $(r=-0.52, p$ $<0.001)$ and meta-cognition domains $(r=-0.23, p=$ $0.02)$, reflected by higher BRIEF-A $T$-scores. Among the neuropsychological tests, ORTO-15 score was negatively related to CVLT-II intrusion errors $(r=-0.20, p=0.04)$, indicative of more verbal long-term memory errors as ON symptom severity increased.

Hierarchical multiple regression, controlling for Body Dissatisfaction, Perfectionism, and OCI-R total scores, showed that ORTO-15 score contributed unique variance to the Set-Shifting, Emotional Control, Self-monitoring, and Working Memory BRIEF-A subscales (Table 2). ORTO-15 score did not contribute unique variance to any of the CVLT-II variables.

\subsection{Analyses between Groups}

Within the full sample, the 21 participants (20 women) who met the Donini et al. threshold for ON [9] were assigned to the orthorexic group $\left(\mathrm{ON}^{+}\right)$, a prevalence rate comparable to what Ramacciotti et al. (2011) found in their community sample [22]. Another 21 participants (10 women) with the highest ORTO-15 total scores formed a non-orthorexic reference group $\left(\mathrm{ON}^{-}\right)$. Importantly, these groups were comparable in age, education, and estimated intellectual functioning (all $p$-values $=\mathrm{ns}$ ). Although the gender ratio was imbalanced, $\chi^{2}(1)=11.67$, $p<0.001$, the high preponderance of women in the $\mathrm{ON}^{+}$ group is consistent with other reported samples [22,23].

As shown in Table 1, ANOVA revealed group differences in Body Dissatisfaction, $F(1,40)=30.41, p<0.001$, perfectionism, $F(1,40)=7.51, p<0.01$, and total OCD symptoms, $F(1,40)=15.03, p<0.001$. In each instance, the $\mathrm{ON}^{+}$group reported more pathology than the $\mathrm{ON}^{-}$ group. Among performance-based measures, the $\mathrm{ON}^{-}$ group outperformed the $\mathrm{ON}^{+}$group on the WCST in number of correct trials, $F(1,40)=3.95, p<0.05$. Multivariate analysis of variance (MANOVA) with the BRIEF-A Behavior Regulation and Meta-Cognition Indices as dependent measures indicated that the $\mathrm{ON}^{+}$ group endorsed more executive dysfunction than the $\mathrm{ON}^{-}$group, $F(1,40)=18.41, p<0.001$ and $F(1,40)=$ $4.27, p<0.05$ respectively. These differences were present in the specific subscales of Shifting, $F(1,40)=$ 20.28, $p<0.001$, Emotional Control, $F(1,40)=22.33, p$ $<0.001$, Initiating, $F(1,40)=5.28, p<0.05$, Working Memory, $F(1,40)=4.13, p<0.05$, Planning, $F(1,40)=$ 4.46, $p<0.05$, and Task Monitoring, $F(1,40)=5.58, p<$ 0.05 . The degree of reported weakness among these BRIEF-A subscales was within half to one $S D$ above the population norm. Using ANCOVA to control for Body Dissatisfaction, Perfectionism, and OCI-R total scores, of the effects described above, the two groups differed only with regard to total correct trials on the WCST, $F(1,37)=$ $9.9, p<0.005$, with the $\mathrm{ON}^{-}$outperforming the $\mathrm{ON}^{+}$ group.

\section{DISCUSSION}

This study is the first neuropsychological evaluation of ON. Given reports of symptom overlap between $\mathrm{ON}, \mathrm{AN}$, and OCD, we expected that cognitive performance would covary inversely with $\mathrm{ON}$ symptom severity and that $\mathrm{ON}^{-}$individuals would outperform $\mathrm{ON}^{+}$individuals in one or more cognitive domains typically affected in $\mathrm{AN}$ and OCD. Across the entire sample, ON severity was negatively associated with executive functioning as assessed via self-report (BRIEF-A). Additional analysis of extreme groups revealed that $\mathrm{ON}^{+}$individuals reported more broadband executive dysfunction on the BRIEF-A and had worse performance on the WCST (number of total correct trials).

As expected, ON, AN, and OCD symptoms were strongly inter-correlated in the full sample, with $\mathrm{ON}^{+}$ individuals endorsing significant AN and OCD symptoms. Given this comorbidity, we reevaluated the above findings to determine if $\mathrm{ON}$ provides unique variance in neuropsychological performance beyond the contributions of AN- and OCD-related variables. Hierarchical regressions in the full sample showed that $\mathrm{ON}$ contributes uniquely to several of the BRIEF-A subscales. ANCOVA indicated that the $\mathrm{ON}^{-}$group outperformed the $\mathrm{ON}^{+}$group on WCST total correct trials. Taken together, it appears that $\mathrm{ON}$ is associated with similar cognitive correlates that are seen at the intersection of $\mathrm{AN}$ and OCD. Although some of the cognitive effects found in this sample are likely better explained by some combination of AN and OCD symptoms, an important finding is that ON symptoms are independently associated with aspects of executive functioning: set-shifting, selfmonitoring, and working memory (WM). Interestingly, these areas of executive functioning represent what would be considered the neuropsychological overlap among OCD and AN profiles.

Set-shifting involves flexible problem-solving and the 
Table 1. Descriptive statistics and Pearson correlations.

\begin{tabular}{|c|c|c|c|c|c|c|}
\hline \multirow{2}{*}{$\frac{\text { Scale/Test }}{\text { Variable }}$} & \multicolumn{3}{|c|}{ Extreme groups } & \multicolumn{2}{|c|}{ Entire sample } & \multirow{2}{*}{$\begin{array}{c}\text { Correlations } \\
\text { ORTO-15 }\end{array}$} \\
\hline & $\mathrm{ON}^{+}$ & $\mathrm{ON}^{-}$ & Partial $\eta^{2}$ & $M$ & $S D$ & \\
\hline ORTO-15 total score & 34.7 & 54.7 & $0.91^{* * *}$ & 45.3 & 7.3 & ----- \\
\hline OCI-R total score & 23.5 & 10 & $0.27^{* * *}$ & 15.7 & 12.4 & $-0.41^{* * *}$ \\
\hline \multicolumn{7}{|l|}{ Eating Disorders Inventory, Second Edition } \\
\hline Perfectionism score & 27.7 & 22.9 & $0.16^{* *}$ & 24.5 & 5.7 & $-0.30^{* *}$ \\
\hline Body Dissatisfaction score & 40.6 & 20.2 & $0.43^{* * *}$ & 29.9 & 12.7 & $-0.51^{* * *}$ \\
\hline \multicolumn{7}{|l|}{ Wide Range Achievement Test, Fourth Edition } \\
\hline Reading subtest SS & 118.2 & 116.6 & 0.01 & 118.5 & 9.4 & -0.04 \\
\hline \multicolumn{7}{|l|}{ Behavior Rating Inventory of Executive Functions } \\
\hline Inhibition $T$-score & 56.3 & 52.6 & 0.04 & 52.5 & 10.1 & $-0.28^{* *}$ \\
\hline Set-Shifting $T$-score & 59.4 & 52.6 & $0.34^{* * *}$ & 52.8 & 9.1 & $-0.46^{* * *}$ \\
\hline Emotional Control $T$-score & 55.4 & 43.7 & $0.36^{* * *}$ & 47.8 & 9.1 & $-0.54^{* * *}$ \\
\hline Self-Monitoring $T$-score & 50.6 & 45.1 & 0.08 & 46.6 & 8.9 & $-0.35^{* * *}$ \\
\hline Task Initiation $T$-score & 52.7 & 46.8 & $0.12^{*}$ & 48.7 & 9.3 & -0.16 \\
\hline Working Memory $T$-score & 58 & 51.5 & $0.09^{*}$ & 54.1 & 10.3 & $-0.29^{* *}$ \\
\hline Planning/Organization $T$-score & 55.8 & 49.7 & $0.10^{*}$ & 51.1 & 9.6 & $-0.22^{*}$ \\
\hline Task Monitoring $T$-score & 58.5 & 51.3 & $0.12^{*}$ & 54.2 & 9.7 & $-0.26^{* *}$ \\
\hline Organization of Materials $T$-score & 46.2 & 46.2 & 0.00 & 46.9 & 9.4 & -0.01 \\
\hline \multicolumn{7}{|l|}{ California Verbal Learning Test, Second Edition } \\
\hline Acquisition $T$-score & 54.1 & 53.2 & 0.00 & 53.9 & 10.3 & -0.02 \\
\hline Short Delay Free Recall $z$-score & 0.6 & 0.1 & 0.07 & 0.3 & 1 & -0.16 \\
\hline Short-Delay Cued Recall $z$-score & 0.5 & 0.0 & 0.07 & 0.3 & 1 & -0.15 \\
\hline Long-Delay Free Recall $z$-score & 0.4 & -0.2 & 0.06 & 0.2 & 1 & -0.12 \\
\hline Long-Delay Cued Recall $z$-score & 0.5 & -0.2 & 0.07 & 0.2 & 1 & -0.19 \\
\hline Recognition Score $z$-score & 0.5 & 0.5 & 0.02 & -0.3 & 0.7 & -0.01 \\
\hline Semantic Clustering Index $z$-score & 0.6 & 0.7 & 0.00 & 0.9 & 1.6 & 0.02 \\
\hline Serial Clustering Index $z$-score & 0.2 & 0.1 & 0.00 & -0.1 & 1.5 & -0.03 \\
\hline \multicolumn{7}{|l|}{ Wisconsin Card Sorting Test } \\
\hline Total Number of Trials & 82.2 & 87 & 0.04 & 83.7 & 13.4 & 0.09 \\
\hline Total Correct Responses & 67.5 & 71.3 & $0.10^{*}$ & 68.6 & 6.5 & 0.1 \\
\hline Learning-to-Learn Index $z$-score & 0.2 & -0.7 & 0.02 & -0.1 & 2.6 & -0.12 \\
\hline \multicolumn{7}{|l|}{ Delis-Kaplan Executive Function System } \\
\hline Color-Word: Inhibition SS & 11.4 & 12 & 0.02 & 11.6 & 2 & 0.06 \\
\hline Color-Word: Inhibition/Switching SS & 12.1 & 11.8 & 0.01 & 11.6 & 1.8 & -0.08 \\
\hline Trail Making: Number-Letter Switching SS & 10.4 & 10.8 & 0.01 & 10.8 & 2 & 0.14 \\
\hline Verbal Fluency: Letter Fluency SS & 13.5 & 12.4 & 0.04 & 13.7 & 3 & -0.05 \\
\hline Verbal Fluency: Category Fluency SS & 13.8 & 13.6 & 0.00 & 13.3 & 2.9 & -0.03 \\
\hline Verbal Fluency: Category Switching SS & 14.5 & 13.7 & 0.02 & 13.8 & 2.9 & -0.16 \\
\hline Verbal Fluency: Category Switching Accuracy SS & 14.5 & 13.9 & 0.01 & 14 & 2.5 & -0.11 \\
\hline Design Fluency Total SS & 14.7 & 14.7 & 0.00 & 14.5 & 2.7 & -0.1 \\
\hline
\end{tabular}

Note. $\mathrm{ON}^{+}=$orthorexic group; $\mathrm{ON}^{-}=$non-orthorexic group; ORTO-15 = Orthorexia Scale; OCI-R = Obsessive Compulsive Inventory, Revised; SS = scaled score. Partial $\eta^{2}$ values indicate effect size. Pearson correlations are two-tailed. ${ }^{*} p \leq 0.05 ;{ }^{* *} p \leq 0.01 ;{ }^{* * *} p \leq 0.001$. 
Table 2. Hierarchical multiple regressions.

\begin{tabular}{lcccc}
\hline Variable & $R^{2}$ & $F$ & $\Delta R^{2}$ & $F$ change \\
\hline BRIEF-A Set-Shifting & 0.37 & $14.07^{* * *}$ & 0.04 & $5.34^{*}$ \\
BRIEF-A Emotional Control & 0.37 & $13.69^{* * *}$ & 0.09 & $13.15^{* * *}$ \\
BRIEF-A Self-Monitoring & 0.14 & $3.91^{* *}$ & 0.08 & $9.11^{* *}$ \\
BRIEF-A Working Memory & 0.22 & $6.83^{* * *}$ & 0.05 & $5.55^{*}$ \\
\hline
\end{tabular}

Note. Predictors in the full regression model, which provided the $R^{2}$ values, were ED-2 Perfectionism, ED-2 Body Dissatisfaction, OCI-R total score, and ORTO-15 total score. The $\Delta R^{2}$ values show the portion of variance accounted for by ORTO-15 total score when added last. BRIEF-A = Behavior Rating Inventory of Executive Functions, Adult version. ${ }^{*} p \leq 0.05 ;{ }^{* *} p \leq 0.01 ;{ }^{* * *} p \leq 0.001$.

ability to move freely from one situation to another as needed. Certain orthorexic behaviors are consistent with a weakness in set-shifting. For example, orthorexic individuals typically develop specific rules about food selection (e.g., cannot purchase foods with preservatives), preparation (e.g., must eat only raw foods), and consumption (e.g., must eat foods alone or in set combinations). As these rules increase in number or complexity, the orthorexic individual devotes more time and energy to satisfy these rules and may limit exposure to situations in which rule-following is difficult (e.g., eating at restaurants, sharing a meal with others); with a decline in exposure to varied, stimulating daily activities may come further decline in set-shifting skills through lack of practice. Behavioral and cognitive inflexibility are also well documented in AN [5] and OCD [24].

Self-monitoring, particularly as construed by the BRIEF-A, references the ability to attend to one's behavior in social contexts by tracking the effects of one's behavior on others. Much of the behavioral syndrome of $\mathrm{ON}$ is derivative of an extreme focus on the self, chiefly bodily health and purity. Such a high degree of self-focus may come at the expense of attention to others, as seems to be the case in AN and OCD. Anorexics demonstrate high rates of self-focused attention such that they privilege inward perceptual, cognitive, and emotional information as opposed to external information [25], a cognitive bias that could explain the concomitant weakness in sustained attention to environmental cues [26]. Anorexics also demonstrate a bias in attending to images of their own body relative to images of other bodies [27]. Likewise, individuals with OCD demonstrate elevated cognitive self-consciousness (i.e., the tendency to be aware of and to monitor one's thoughts) [28], elevated self-reflectiveness (i.e., self-preoccupation and rumination) [29], and reduced capacity for theory of mind [30].

WM refers to the ability to hold information on-line for brief periods of time in order to complete a task. As WM is a depletable resource, simultaneous processing of task-irrelevant stimuli, particularly negative emotional distracters, competes with ongoing operations [31]. From this perspective, it is possible that reduced WM capacity in $\mathrm{ON}$ is secondary to a preoccupation with food-related thoughts and images. Similar findings have been reported in the eating disorders literature. For example, WM dysfunction has been identified in anorexic patients [32], with specific deficits in the central executive and visuospatial sketch pad subsystems [33]. Furthermore, there is growing evidence that food-related thoughts reduce WM capacity in individuals who are restricting their diets for whatever reason $[34,35]$. While the literature examining memory performance in OCD is quite mixed [36], a recent meta-analysis [37] implicates WM as the link between executive dysfunction and long-term memory impairment. Specifically, Harkin and Kessler [37] pinpoint the episodic buffer [38], which permits multimodal, temporarily-integrated binding of environmental features, as the element of WM that is deficient in $\mathrm{OCD}$, the idea being that intrusive thoughts interfere with ongoing episodic binding.

As a correlational study, we are unable to comment on the temporal ordering of the neuropsychological weaknesses and ON behaviors. It is unknown whether setshifting, self-monitoring, and WM dysfunction precede $\mathrm{ON}$, thereby serving as developmental risk factors, or whether these variables are cognitive sequelae. Similar issues concerning the temporal relationships of neuropsychological functioning in AN and OCD are still debated $[39,40]$. Prospective, longitudinal neuropsychological studies are needed to address questions of causal attribution in ON. Such designs are also an ideal way to explore the degree and nature of interconnection between $\mathrm{ON}, \mathrm{AN}$, and OCD traits. What makes this a complicated proposition is that the temporal precedence of $\mathrm{AN}$ and OCD, when these two conditions are comorbid, is itself unclear [41]. Some argue that AN and OCD belong to the same obsessive-compulsive spectrum [42] and share common etiopathogenic roots at the genetic [43] and neurochemical levels [44]. Should ON fit into what may be a complex phenotype, future research should assess whether set-shifting, self-monitoring, and WM weaknesses are cognitive endophenotypes that fill an explanatory gap between genes and behavior.

Although important as the first neuropsychological analysis of $\mathrm{ON}$, the current findings must be considered in the context of the study's limitations. First, even 
though the ORTO-15 is the sole measure in existence to assess the ON construct, its reliability and validity have not been thoroughly tested. In the present sample, the internal consistency of the ORTO-15 was sufficiently high, but little is known about test-retest reliability and indices of construct and predictive validity across populations. Second, this study was conducted with a nonclinical, young adult sample. Such samples are common in the OCD and AN literature, as the age of onset and base rates of these disorders are such that there is enough symptom endorsement to permit dimensional and categorical analysis of the data. Although variance in ORTO15 score in the present sample suggests that a college student population is a reasonable one in which to study $\mathrm{ON}$, it is worth replicating this study in unselected community samples with a broader age range as well as in clinical samples with patients diagnosed with eating disorders. Third, even though our sample was screened with the M.I.N.I. to exclude those with diagnosable DSM-IV pathology, by not including measures of depression and anxiety, we cannot determine the extent to which fluctuations in these variables influenced neuropsychological performance. Subsequent studies that examine the unique contributions of ON to cognition should assess mood state at the time of testing. Furthermore, future cognitive studies of ON should include additional measures of set-shifting, self-monitoring, and WM, whether these come from standardized clinical batteries or from experimental paradigms. Even though the WCST is considered a "gold standard" of executive function testing [16], it does not permit clean separation of the constituent functions. Moreover, it appears that the executive functions needed to perform the WCST may vary as a function of age [45], making it additionally imperative that future neuropsychological studies of $\mathrm{ON}$ be conducted in samples with a broad age range.

\section{REFERENCES}

[1] Bratman, S. and Knight, D. (2000) Health food junkies. Broadway, New York.

[2] Fidan, T., Ertekin, V., Isikay, S. and Kirpinar, I. (2010) Prevalence of orthorexia among medical students in Erzurum, Turkey. Comprehensive Psychiatry, 51, 49-54. doi:10.1016/j.comppsych.2009.03.001

[3] Donini, L.M., Marsili, D., Graziani, M.P., Imbriale, M. and Cannella, C. (2004) Orthorexia nervosa: A preliminary study with a proposal for diagnosis and an attempt to measure the dimension of the phenomenon. Eating and Weight Disorders, 9, 151-157.

[4] Kingston, K., Szmukler, G., Andrewes, D., Tress, B. and Desmond, P. (1996) Neuropsychological and structural brain changes in anorexia nervosa before and after refeeding. Psychological Medicine, 26, 15-28.

doi:10.1017/S0033291700033687
[5] Steinglass, J.E., Walsh, B. and Stern, Y. (2006) Set shifting deficit in anorexia nervosa. Journal of the International Neuropsychological Society, 12, 431-435. doi:10.1017/S1355617706060528

[6] Fontenelle, L.F., Mendlowicz, M.V., Mattos, P. and Versiani, M. (2006) Neuropsychological findings in obsessive-compulsive disorder and its potential implications for treatment. Current Psychiatry Reviews, 2, 11-26. doi:10.2174/157340006775101454

[7] Chamberlain, S.R., Blackwell, A.D., Fineberg, N.A., Robbins, T.W. and Sahakian, B.J. (2005) The neuropsychology of obsessive compulsive disorder: The importance of failures in cognitive and behavioural inhibition as candidate endophenotypic markers. Neuroscience and Biobehavioral Reviews, 29, 399-419. doi:10.1016/j.neubiorev.2004.11.006

[8] Sheehan, D.V., Lecrubier, Y., Harnett-Sheehan, K., et al. (1998) The Mini International Neuropsychiatric Interview (M.I.N.I.): The Development and validation of a structured diagnostic psychiatric interview for DSM-IV and ICD-10. Journal of Clinical Psychiatry, 59, 22-23.

[9] Donini, L.M., Marsili, D., Graziani, M.P., Imbriale, M. and Cannella, C. (2005) Orthorexia nervosa: Validation of a diagnosis questionnaire. Eating and Weight Disorders, 10, 28-32.

[10] Garner, D.M., Olmstead, M.P. and Polivy, J. (1983) Development and validation of a multidimensional eating disorder inventory for anorexia nervosa and bulimia. International Journal of Eating Disorders, 2, 15-34. doi:10.1002/1098-108X(198321)2:2<15::AID-EAT22600 20203>3.0.CO;2-6

[11] Foa, E.B., Huppert, J.D., Leiberg, S., et al. (2002) The Obsessive-Compulsive Inventory: Development and validation of a short version. Psychological Assessment, 14, 485-496. doi:10.1037/1040-3590.14.4.485

[12] Wilkinson, G.S. and Robertson, G.J. (2006) Wide range achievement test: Professional manual. Psychological Assessment Resources, Florida.

[13] Roth, R.M., Isquith, P.K. and Gioia, G.A. (2005) Behavior rating inventory of executive function-adult version. Psychological Assessment Resources, Florida.

[14] Delis, D.C., Kaplan, E. and Kramer, J.H. (2001) DelisKaplan Executive Function System. Pearson Assessments, Texas.

[15] Delis, D.C., Kramer, J., Kaplan, E. and Ober, B. (2000) California Verbal Learning Test. 2nd Edition, Pearson Assessments, Texas.

[16] Grant, D.A. and Berg, E.A. (2003) Wisconsin Card Sorting Test. Psychological Assessment Resources, Florida.

[17] McInerney-Ernst, E.M. (2012) Orthorexia nervosa: Real construct or newest social trend? Doctoral Dissertation, ProQuest Dissertations and Theses Database (AAI3465913).

[18] Gulker, M.G., Laskis, T.A. and Kuba, S.A. (2001) Do excessive exercisers have a higher rate of obsessivecompulsive symptomatology? Psychology, Health and Medicine, 6, 387-398.

[19] Abbate-Daga, G., Piero, A., Gramaglia, C., Gandione, M. 
and Fassino, S. (2007) An attempt to understand the paradox of anorexia nervosa without drive for thinness. Psychiatry Research, 149, 215-221. doi:10.1016/i.psychres.2005.10.017

[20] Ramaciotti, C.E., Dell'Osso, L., Paoli, R.A., Ciapparelli, A., Coli, E., Kaplan, A.S. and Garfinkel, P. (2002) Characteristics of eating disorder patients without a drive for thinness. International Journal of Eating Disorders, 32, 206-212. doi:10.1002/eat.10067

[21] Rothman, K.J. (1990) No adjustments are needed for multiple comparisons. Epidemiology, 1, 43-46. doi:10.1097/00001648-199001000-00010

[22] Ramacciotti, C.E., Perrone, P., Coli, E., et al. (2011) Orthorexia nervosa in the general population: A preliminary screening using a self-administered questionnaire (ORTO-15). Eating and Weight Disorders, 16, 127-130.

[23] Bosi, A.T.B., Camur, D. and Güler, C. (2007) Prevalence of orthorexia nervosa in resident medical doctors in the faculty of medicine. Appetite, 49, 661-666. doi:10.1016/j.appet.2007.04.007

[24] Gu, B.M., Park, J.Y., Kang, D.H., et al. (2008) Neural correlates of cognitive inflexibility during task-switching in obsessive-compulsive disorder. Brain: Journal of Neurology, 131, 155-164. doi:10.1093/brain/awm277

[25] Sproch, L. (2011) The effect of self-focused attention on female Body Dissatisfaction in subclinical anorexia nervosa and subclinical binge eating disorder. Doctoral dissertation, ProQuest Dissertations and Theses Database (AAI3448573).

[26] Fowler, L., Blackwell, A., Jaffa, A., et al. (2006) Profile of neurocognitive impairments associated with female in-patients with anorexia nervosa. Psychological Medicine, 36, 517-527. doi:10.1017/S0033291705006379

[27] Blechert, J., Ansorge, U. and Tuschen-Caffier, B. (2010) A body-related dot-probe task reveals distinct attentional patterns for bulimia nervosa and anorexia nervosa. Journal of Abnormal Psychology, 119, 575-585. doi:10.1037/a0019531

[28] Kikul, J., Van Allen, T.S. and Exner, C. (2012) Underlying mechanisms of verbal memory deficits in obsessive-compulsive disorder and major depression-The role of cognitive self-consciousness. Journal of Behavior Therapy and Experimental Psychiatry, 43, 863-870. doi:10.1016/j.jbtep.2011.12.009

[29] O'Kearney, R. and Nicholson, C. (2008) Can a theory of mind disruption help explain OCD related metacognitive disturbances? Behavior Change, 25, 55-70. doi:10.1375/bech.25.2.55

[30] Grisham, J.R., Henry, J.D., Williams, A.D. and Bailey, P.E. (2009) Socioemotional deficits associated with obsessive-compulsive symptomatology. Psychiatry Research, 175, 256-259. doi:10.1016/j.psychres.2009.01.028

[31] Anticevic, A., Repovs, G. and Barch, D.M. (2010) Resisting emotional interference: Brain regions facilitating working memory performance during negative distraction. Cognitive, Affective and Behavioral Neuroscience, 10, 159-173. doi:10.3758/CABN.10.2.159

[32] Castro-Fornieles, J., Caldú, X. Andrés-Perpiñá, S., et al.
(2010) A cross-sectional and follow-up functional MRI study with a working memory task in adolescent anorexia nervosa. Neuropsychologia, 48, 4111-4116. doi:10.1016/j.neuropsychologia.2010.10.003

[33] Kemps, E., Tiggemann, M., Wade, T., Ben-Tovim, D. and Breyer, R. (2006) Selective working memory deficits in anorexia nervosa. European Eating Disorders Review, 14, 97-103. doi:10.1002/erv.685

[34] Kemps, E. and Tiggemann, M. (2005) Working memory performance and preoccupying thoughts in female dieters: Evidence for a selective central executive impairment. British Journal of Clinical Psychology, 44, 357-366. doi:10.1348/014466505X35272

[35] Vreugdenburg, L., Bryan, J. and Kemps, E. (2003) The effect of self-initiated weight-loss dieting on working memory: The role of preoccupying cognitions. Appetite, 41, 291-300. doi:10.1016/S0195-6663(03)00107-7

[36] Hermans, D., Engelen, U., Grouwels, L., Joos, E., Lemmens, J. and Pieters, G. (2008) Cognitive confidence in obsessive-compulsive disorder: Distrusting perception, attention and memory. Behavior Research and Therapy, 46, 98-113. doi:10.1016/j.brat.2007.11.001

[37] Harkin, B. and Kessler, K. (2011) The role of working memory in compulsive checking and OCD: A systematic classification of 58 experimental findings. Clinical Psychology Review, 31, 1004-1021. doi:10.1016/j.cpr.2011.06.004

[38] Baddeley, A.D. (2000) The episodic buffer: A new component of working memory? Trends in Cognitive Science, 4, 417-423. doi:10.1016/S1364-6613(00)01538-2

[39] Holliday, J., Tchanturia, K., Landau, S., Collier, D. and Treasure, J. (2005) Is impaired set-shifting an endophenotype of anorexia nervosa? American Journal of Psychiatry, 162, 2269-2275. doi:10.1176/appi.ajp.162.12.2269

[40] Viswanath, B., Reddy, Y.C.J., Kumar, K.J., Kandavel, T. and Chandrashekar, C.R. (2009) Cognitive endophenotypes in OCD: A study of unaffected siblings of probands with familial OCD. Progress in Neuro-Psychopharmacology and Biological Psychiatry, 33, 610-615. doi:10.1016/j.pnpbp.2009.02.018

[41] Buckner, J.D., Silgado, J. and Lewinsohn, P.M. (2010) Delineation of differential temporal relations between specific eating and anxiety disorders. Journal of Psychiatric Research, 44, 781-787. doi:10.1016/j.jpsychires.2010.01.014

[42] Lochner, C. and Stein, D.J. (2006) Does work on obsessive-compulsive spectrum disorders contribute to understanding the heterogeneity of obsessive-compulsive disorder? Progress in Neuro-Psychopharmacological and Biological Psychiatry, 30, 353-361. doi:10.1016/j.pnpbp.2005.11.004

[43] Cavallini, M.C., Bertelli, S., Chiapparino, D., Riboldi, S. and Bellodi, L. (2000) Complex segregation analysis of obsessive-compulsive disorder in 141 families of eating disorder probands, with and without obsessive-compulsive disorder. American Journal of Medical Genetics, 96, 384-391.

doi:10.1002/1096-8628(20000612)96:3<384::AID-AJM 
G28>3.0.CO;2-P

[44] Barbarich, N. (2002) Is there a common mechanism of serotonin dysregulation in anorexia nervosa and obsessive compulsive disorder? Eating and Weight Disorders, 7, 221-231.
[45] Gamboz, N., Borella, E. and Brandimonte, M.A. (2009) The role of switching, inhibition and working memory in older adults' performance in the Wisconsin Card Sorting Test. Aging, Neuropsychology and Cognition, 16, 260284. doi: $10.1080 / 13825580802573045$ 